\title{
An unusual case of sodium citrate-dependent artifactual platelet count
}

\author{
FRANCESCO DIMA $^{1}$, GIAN LUCA SALVAGNO ${ }^{1}$, ELISA DANESE ${ }^{1}$, DINO VENERI ${ }^{2}$, \\ GIUSEPPE LIPPI ${ }^{1, *}$
}

\author{
${ }^{1}$ Section of Clinical Biochemistry, University Hospital of Verona, Verona, Italy \\ ${ }^{2}$ Hematology Section, Department of Medicine, University of Verona, Verona, Italy \\ *Corresponding author: Prof. Giuseppe Lippi; Section of Clinical Biochemistry, University Hospital of Verona, Piazzale LA Scuro, 37134 Verona, \\ Italy; Phone: +39045 8124308; Fax: +39 045 916735; E-mail: giuseppe.lippi@univr.it
}

(Received: February 4, 2019; Accepted: July 24, 2019)

\begin{abstract}
Background: Ethylenediaminetetraacetic acid (EDTA)-dependent pseudothrombocytopenia is a rare phenomenon. Spurious pseudothrombocytopenia has also been described in other circumstances, while artifactual platelet count in whole blood samples anticoagulated with sodium citrate is an exceptional occurrence. Case report: In this study, we describe the case of a 44-year-old ostensibly healthy woman who attended the local outpatient clinic for routine laboratory testing, including platelet count in EDTA and sodium citrate, for suspected artifactual pseudothrombocytopenia previously identified in another center. The results of hematological testing on both specimens were essentially normal, except for mild anemia. Nevertheless, the platelet number was $425 \times 10^{9} / \mathrm{L}$ in $\mathrm{K}_{2}$ EDTA and $266 \times 10^{9} / \mathrm{L}\left(293 \times 10^{9} / \mathrm{L}\right.$ after correcting for sample dilution) in sodium citrate, respectively. Microscopic revision of blood smears revealed the presence of platelet aggregates and satellitism only in the sodium citrate specimen. Conclusion: Unlike previous occasional reports of concomitant EDTA- and sodium citrate-dependent pseudothrombocytopenia, we first describe a paradigmatic case of artifactual platelet count attributable to platelet clumping and satellitism, exclusively developing in blood anticoagulated with sodium citrate.
\end{abstract}

Keywords: platelets, pseudothrombocytopenia, EDTA, sodium citrate, case report

\section{Introduction}

Laboratory hematology is an essential part of the diagnostic approach and clinical decision-making in patients with hematological disturbances [1]. Although many technological advances occurred over the past decades have made it possible to considerably expand volume and complexity of in vitro hematological investigations $[2,3]$, the complete blood cell count $(\mathrm{CBC})$ remains the cornerstone for screening, diagnosis, and therapeutic monitoring of the vast majority of blood cells disorders. The CBC is typically performed on ethylenediaminetetraacetic acid (EDTA)-anticoagulated venous blood specimens, since this additive irreversibly sequestrate ionized calcium $\left(\mathrm{Ca}^{2+}\right)$, thus permanently inhibiting blood coagulation and enabling a better stability of anticoagulated blood for purposes of cell enumeration and sizing [4]. The dipotassium salt of this additive (i.e., $\mathrm{K}_{2}$ EDTA) has been identified as the anticoagulant of choice for blood cell enumeration and sizing by the International Council for Standardization in Haematology [5], since it avoids dilution effects and has a lower impact on erythrocyte and platelet size compared to other additives. Nevertheless, the use of $\mathrm{K}_{2}$ EDTA has also been associated with a number of drawbacks, the most important of which is indeed EDTA-dependent pseudothrombocytopenia [6, 7]. Overall, EDTA-dependent pseudothrombocytopenia is considered as a relatively rare phenomenon, occurring in approximately $0.1 \%-0.2 \%$ venous blood specimens. Platelet aggregation in EDTAanticoagulated samples is mostly attributable to the presence of EDTA-dependent antiplatelet antibodies, which are reactive between 0 and $4{ }^{\circ} \mathrm{C}$ and recognize activation antigens [7]. Once the antibodies bind to platelet surface receptors, in vitro platelet aggregation is triggered, thus causing variable degrees of platelet agglutination and/or aggregation. Since failure to recognize a spuriously decreased platelet count may derange the clinical reasoning

This is an open-access article distributed under the terms of the Creative Commons Attribution-NonCommercial 4.0 International License, which permits unrestricted use, distribution, and reproduction in any medium for non-commercial purposes, provided the original author and source are credited, a link to the CC License is provided, and changes - if any - are indicated. 
and cause unwarranted clinical consequences, some reliable options have been proposed to overcome this artifactual event. These typically include drawing blood into blood tubes containing different anticoagulants (e.g., sodium citrate, sodium fluoride, lithium or calcium chloride heparin, ammonium oxalate and trisodium citrate pyridoxal 5-phosphate, and Tris). Among these different options, sample collection in $3.2 \%$ buffered sodium citrate is the most practical and thus it is deemed as the most widely used approach. In this study, we describe the case of a patient with artifactual platelet count in sodium citrate.

\section{Case Report}

A 44-year-old ostensibly healthy woman attended the outpatient clinic of the University Hospital of Verona for routine laboratory testing before undergoing in vitro fertilization, including platelet count in two sequentially drawn $\mathrm{K}_{2}$ EDTA and sodium citrate blood tubes (Vacutest Kima, Padova, Italy), for suspected artifactual platelet count previously identified in another center (the original value is unavailable). Hematological testing was performed with Sysmex XN (Sysmex Corporation, Kobe, Japan), which uses a combination of flow cytometry, impedance technique, and/or fluorescence for platelet analysis [8]. The results of CBC performed on both specimens within $30 \mathrm{~min}$ after blood collection were essentially normal (i.e., comprised within the relative reference ranges), except for mild anemia (Table I). Nevertheless, a discrepancy was found between the platelet counts carried out in the two blood samples. More specifically, the platelet number was $425 \times 10^{9} / \mathrm{L}$ in $\mathrm{K}_{2}$ EDTA and $266 \times 10^{9} / \mathrm{L}\left(293 \times 10^{9} / \mathrm{L}\right.$ after correction for sample dilution, i.e., $\sim 30 \%$ lower) in sodium citrate, respectively (Table I). Repeated analyses of the two specimens 2 and $5 \mathrm{~h}$ after collection yielded identical data. In order to rule out that the lower platelet count in sodium citrate could be attributable to a preanalytical error, the various preanalytical steps were accurately verified, so excluding problems of inaccurate blood tube filling, presence of visible clots, and so forth. Peripheral blood smears, stained in May-Grunwald-Giemsa (Carlo Erba, Milano, Italy), were then prepared for identifying possible platelet aggregates, which were not flagged by the hematological analyzer. Notably, the microscopic revision of the blood smears in $\mathrm{K}_{2}$ EDTA and sodium citrate revealed the clear presence of platelet aggregates and satellitism only in the sodium citrate specimen (Fig. 1). This unusual finding was timely communicated to the general practitioner, who decided to avoid followup investigations, since the woman was still healthy and asymptomatic.

\section{Discussion}

A high degree of accuracy and standardization of preanalytical variables in laboratory hematology are essential for obtaining reliable data. Several preanalytical factors, related to specimen collection and handling, are frequent sources of inaccurate test results, thus jeopardizing the quality of testing and the clinical decisionmaking [9].

To date, EDTA-dependent pseudothrombocytopenia is a relatively rare phenomenon, which has been known for nearly 40 years [10]. Other causes of spurious platelet count have been reported, mostly involving samples with presence of cellular debris [11], cryoglobulins [12], and bloodborne pathogens [13]. Sodium citrate-dependent pseudothrombocytopenia is instead a rather unusual, and hence less frequently appreciated, phenomenon in laboratory hematology, since a very limited number of cases have been described to date. Yoshikawa et al. [14] described the case of 65-year-old man, who developed

Table I Results of the complete blood cell count in dipotassium ethylenediaminetetraacetic acid $\left(\mathrm{K}_{2} \mathrm{EDTA}\right)$ and sodium citrate

\begin{tabular}{lccc} 
Hematological parameters & $\mathrm{K}_{2}$ EDTA & Sodium citrate* & Reference range \\
Red blood cells $\left(\times 10^{12} / \mathrm{L}\right)$ & 4.19 & 4.04 & $4.00-5.20$ \\
Hemoglobin $(\mathrm{g} / \mathrm{L})$ & 117 & 114 & $120-160$ \\
Hematocrit & 0.35 & 0.34 & $0.35-0.45$ \\
Mean corpuscular volume (fL) & 85.5 & 84.7 & $80.0-99.0$ \\
Mean corpuscular hemoglobin $(\mathrm{pg})$ & 27 & 28 & $26-34$ \\
Red blood cell distribution width $(\%)$ & 13.6 & 13.8 & $11.5-15.0$ \\
White blood cells $\left(\times 10^{9} / \mathrm{L}\right)$ & 6.74 & 6.78 & $4.50-11.00$ \\
Platelets $\left(\times 10^{9} / \mathrm{L}\right)$ & 425 & 293 & $150-400$ \\
Mean platelet volume $(\mathrm{fL})$ & 9.7 & 9.5 & $9.6-12.9$ \\
\hline
\end{tabular}

${ }^{*}$ Values of cell counts were corrected for sample dilution 

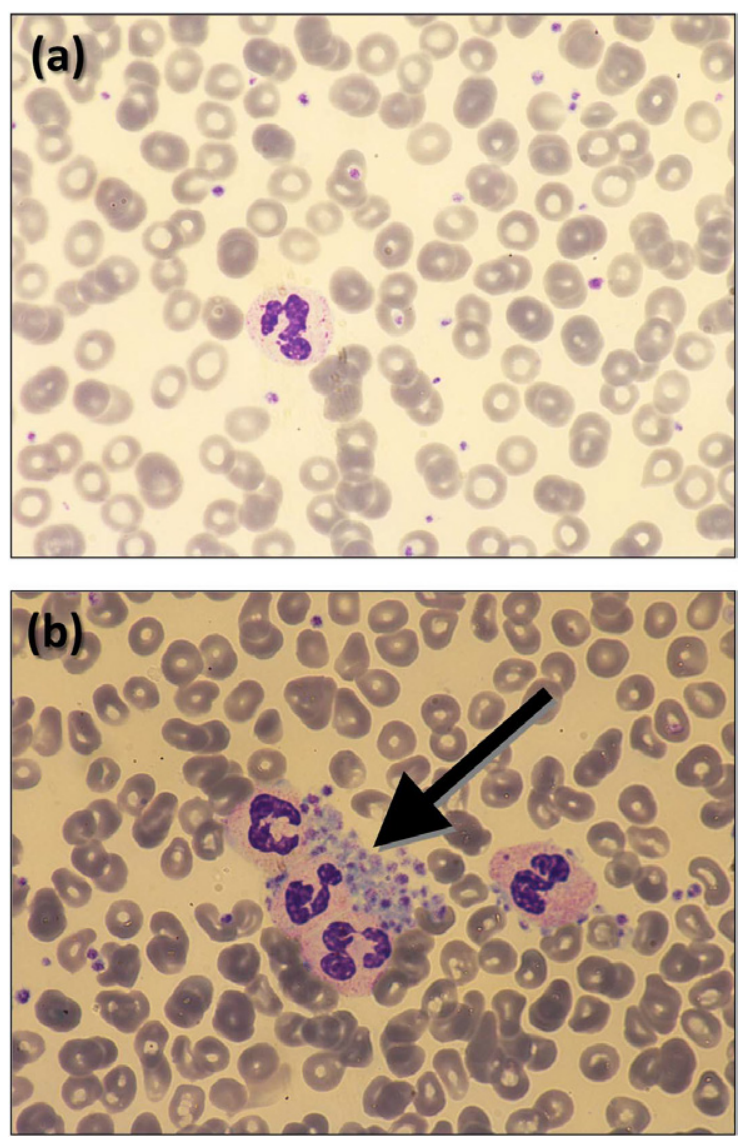

Fig. 1. Blood smears at $100 \times$ magnification of venous blood collected in (a) dipotassium ethylenediaminetetraacetic acid $\left(\mathrm{K}_{2} \mathrm{EDTA}\right)$ and $(\mathrm{b})$ sodium citrate. The black arrow indicates the presence of platelet aggregates and satellitism in the sodium citrate whole blood sample

both EDTA- and sodium citrate-dependent pseudothrombocytopenia after undergoing transcatheter arterial embolization for a hepatocellular carcinoma. However, no clear explanation was given to justify this unusual finding. In an ensuing article, Salama [15] described the cases of two patients with autoimmune thrombocytopenia, whose blood samples developed both EDTAand sodium citrate-dependent pseudothrombocytopenia 20-30 min after blood drawing. Notably, spurious platelet aggregation was found to be probably triggered by the presence of antiplatelet factor 4 (PF4) IgM antibodies. More recently, Kovacs et al. [16] reported the case of an elderly patient, who displayed pseudothrombocytopenia in whole blood samples collected with a vast array of additives (i.e., EDTA, sodium citrate, lithium heparin, disodium oxalate, and hirudin).

Unlike these previous reports, our case has peculiar and paradigmatic features, whereby artifactual platelet count could be attributed to platelet clumping in association with platelet satelittism, but only in the whole blood sample drawn in $3.2 \%$ sodium citrate and not in that collected with $\mathrm{K}_{2} \mathrm{EDTA}$. Since the $\mathrm{CBC}$ is not routinely performed in whole blood anticoagulated with sodium citrate, it is not surprising that no similar cases have earlier been published to the best of our knowledge.

\section{Conclusions}

Artifactual (i.e., spurious) platelet agglutination is often caused by agglutinating antibodies, which bind to platelet surface glycoproteins, especially to PF4 and glycoprotein IIb/IIIa [7]. Platelet satellitism recognizes a similar pathway, since it may also be triggered by antiplatelet antibodies activated by the presence of an additive (typically EDTA), which induces alterations in surface proteins of both platelets and leukocytes, thus catalyzing bridge formation between the two blood cells [17]. It is hence conceivable that platelet agglutination and satellitism in our sample were likely attributable to the presence of some unrecognized antiplatelet antibodies, whose binding to surface platelet receptors would only be triggered by sodium citrate and not by EDTA. We also point out the importance of conventional optical microscopic analysis. No flags or alarms were generated by the hematological analyzer during sample analysis, so that manual revision of blood smears was essential for identifying this unusual phenomenon in our case report, thus preventing potential diagnostic errors.

$$
* * *
$$

Funding sources: No financial support was received for this study.

Authors' contribution: All authors agreed to be accountable for all aspects of the work and ensuring accuracy and integrity and approved the final version of this manuscript.

Conflict of interest: The authors declare no competing interests.

\section{References}

1. Buoro S, Lippi G: Harmonization of laboratory hematology: A long and winding journey. Clin Chem Lab Med 56, 1575-1578 (2018)

2. Olsen K: The first 110 years of laboratory automation: Technologies, applications, and the creative scientist. J Lab Autom 17, 469-480 (2012)

3. Lippi G, Plebani M: Recent developments and innovations in red blood cells diagnostics. J Lab Precis Med 3, 68 (2018)

4. Banfi G, Salvagno GL, Lippi G: The role of ethylenediamine tetraacetic acid (EDTA) as in vitro anticoagulant for diagnostic purposes. Clin Chem Lab Med 45, 565-576 (2007)

5. International Council for Standardization in Haematology: Recommendations of the International Council for Standardization in Haematology for ethylenediaminetetraacetic acid anticoagulation of blood for blood cell counting and sizing. International Council for Standardization in Haematology: Expert panel on cytometry. Am J Clin Pathol 100, 371-372 (1993)

6. Lippi G, Guidi G, Nicoli M: Platelet count in EDTA-dependent pseudothrombocytopenia. Eur J Haematol 56, 112-113 (1996) 
Dima et al.

7. Lippi G, Plebani M: EDTA-dependent pseudothrombocytopenia: Further insights and recommendations for prevention of a clinically threatening artifact. Clin Chem Lab Med 50, 1281-1285 (2012)

8. Buoro S, Seghezzi M, Manenti B, Pacioni A, Carobene A, Ceriotti F, Ottomano C, Lippi G: Biological variation of platelet parameters determined by the Sysmex XN hematology analyzer. Clin Chim Acta 470, 125-132 (2017)

9. Lippi G, Becan-McBride K, Behúlová D, Bowen RA, Church S, Delanghe J, Grankvist K, Kitchen S, Nybo M, Nauck M, Nikolac N, Palicka V, Plebani M, Sandberg S, Simundic AM: Preanalytical quality improvement in quality we trust. Clin Chem Lab Med 51, 229-241 (2013)

10. Pegels JG, Bruynes EC, Engelfriet CP, von dem Borne AE: Pseudothrombocytopenia: An immunologic study on platelet antibodies dependent on ethylene diamine tetra-acetate. Blood 59, 157-161 (1982)

11. van der Meer W, MacKenzie MA, Dinnissen JW, de Keijzer MH: Pseudoplatelets: A retrospective study of their incidence and interference with platelet counting. J Clin Pathol 56, 772-774 (2003)

12. Buoro S, Manenti B, Seghezzi M, Moioli V, Bagorria M, Callegaro A, Ottomano C, Lippi G: Abnormal scattergrams and cell population data generated by fully automated hematological analyzers: New tools for screening malaria infection? Int J Lab Hematol 40, 326-334 (2018)

13. von Ahsen N, Ehrlich B, Scott CS, Riggert J, Oellerich M: Cryoglobulins interfere with platelet counts by optical and impedance methods but not with the CD61 immunoplatelet count. Clin Chem 47, 1858-1860 (2001)

14. Yoshikawa T, Nakanishi K, Maruta T, Takenaka D, Hirota S, Matsumoto S, Saigo K, Ohno Y, Fujii M, Sugimura K: Anticoagulant-induced pseudothrombocytopenia occurring after transcatheter arterial embolization for hepatocellular carcinoma. Jpn J Clin Oncol 36, 527-531 (2006)

15. Salama A: Autoimmune thrombocytopenia complicated by EDTAand/or citrate-dependent pseudothrombocytopenia. Transfus Med Hemother 42, 345-348 (2015)

16. Kovacs F, Varga M, Pataki Z, Rigo E: Pseudothrombocytopenia with multiple anticoagulant sample collection tubes. Interv Med Appl Sci 8, 181-183 (2016)

17. Chakrabarti I: Platelet satellitism: A rare, interesting, in vitro phenomenon. Indian J Hematol Blood Transfus 30, 213-214 (2014) 